\title{
Study on the Mechanism of Danggui-Chuanxiong Herb Pair on Treating Thrombus through Network Pharmacology and Zebrafish Models
}

Mengqi Zhang, ${ }^{1,2, \|}$ Peihai Li, ${ }^{1,2, \|}$ Shanshan Zhang, ${ }^{1,2}$ Xuanming Zhang, ${ }^{1,2}$ Lizhen Wang, ${ }^{1,2}$ Yun Zhang, ${ }^{1}$ Xiaobin $\mathrm{Li},{ }^{1,3,}$ and Kechun Liu ${ }^{1, *}$

${ }^{1}$ Engineering Research Center of Zebrafish Models for Human Diseases and Drug Screening of Shandong Province, Key Laboratory for Biosensor of Shandong Province, Biology Institute, Qilu University of Technology (Shandong Academy of Sciences), Jinan, 250103, China

${ }^{2}$ State Key Laboratory of Biobased Material and Green Papermaking, Qilu University of Technology, Shandong Academy of Sciences, Jinan, 250353, China

${ }^{3}$ Bioengineering Technology Innovation Center of Shandong Province, Heze, 274000, China

${ }^{*}$ Engineering Research Center of Zebrafish Models for Human Diseases and Drug Screening of Shandong Province, Key Laboratory for Biosensor of Shandong Province, Biology Institute, Qilu University of Technology (Shandong Academy of Sciences), Jinan, Shandong 250103, China

E-mail addresses: lixb@sdas.org (X.-B. Li), liukechun2000@163.com (K.-C. Liu)

" These authors contributed equally to this work. 
Table S1. The detailed information of all compounds in Danggui-Chuanxiong.

\begin{tabular}{|c|c|c|c|c|c|c|c|c|c|c|}
\hline No. & Herb & Molecule Name & CAS & MW & $\mathrm{OB}(\%)$ & Caco-2 & DL & $\mathrm{A} \log \mathrm{P}$ & nHdon & nHacc \\
\hline 1 & A. sinensis (Oliv.) Diels & $(+)$-carvene & $5989-27-5$ & 136.26 & 39.84 & 1.83 & 0.02 & 3.5 & 0 & 0 \\
\hline 2 & A. sinensis (Oliv.) Diels & beta-Selinene & $17066-67-0$ & 204.39 & 24.39 & 1.83 & 0.08 & 4.81 & 0 & 0 \\
\hline 3 & A. sinensis (Oliv.) Diels & scopoletol & $92-61-5$ & 192.18 & 27.77 & 0.71 & 0.08 & 1.62 & 1 & 4 \\
\hline 4 & A. sinensis (Oliv.) Diels & palmitic acid & $67701-02-4$ & 256.48 & 19.3 & 1.09 & 0.1 & 6.37 & 1 & 2 \\
\hline 5 & A. sinensis (Oliv.) Diels & nonanal & $75718-12-6$ & 142.27 & 40.28 & 1.31 & 0.02 & 3.22 & 0 & 1 \\
\hline 6 & A. sinensis (Oliv.) Diels & cymol & $4939-75-7$ & 134.24 & 27.2 & 1.86 & 0.02 & 3.51 & 0 & 0 \\
\hline 7 & A. sinensis (Oliv.) Diels & decanal & $112-31-2$ & 156.3 & 29.81 & 1.34 & 0.02 & 3.68 & 0 & 1 \\
\hline 8 & A. sinensis (Oliv.) Diels & (-)-alpha-Pinene & $7785-26-4$ & 136.26 & 46.25 & 1.85 & 0.05 & 2.87 & 0 & 0 \\
\hline 9 & A. sinensis (Oliv.) Diels & beta-Chamigrene & $18431-82-8$ & 204.39 & 31.99 & 1.82 & 0.08 & 4.71 & 0 & 0 \\
\hline 10 & A. sinensis (Oliv.) Diels & myrcene & $123-35-3$ & 136.26 & 24.96 & 1.84 & 0.02 & 3.69 & 0 & 0 \\
\hline 11 & A. sinensis (Oliv.) Diels & safrol & $94-59-7$ & 162.2 & 45.34 & 1.44 & 0.05 & 2.61 & 0 & 2 \\
\hline 12 & A. sinensis (Oliv.) Diels & p-ocimene & $27400-72-2$ & 136.26 & 15.06 & 1.85 & 0.02 & 3.63 & 0 & 0 \\
\hline 13 & A. sinensis (Oliv.) Diels & moslene & $99-85-4$ & 136.26 & 33.02 & 1.88 & 0.02 & 3.45 & 0 & 0 \\
\hline 14 & A. sinensis (Oliv.) Diels & isoeugenol & $5932-68-3$ & 164.22 & 70.1 & 1.38 & 0.04 & 2.5 & 1 & 2 \\
\hline 15 & A. sinensis (Oliv.) Diels & (L)-alpha-Terpineol & $7785-53-7$ & 154.28 & 46.3 & 1.28 & 0.03 & 2.42 & 1 & 1 \\
\hline 16 & A. sinensis (Oliv.) Diels & o-thymol & $499-75-2$ & 150.24 & 43.28 & 1.58 & 0.03 & 3.24 & 1 & 1 \\
\hline 17 & A. sinensis (Oliv.) Diels & $(+)$-3-carene & $498-15-7$ & 136.26 & 45.2 & 1.84 & 0.04 & 2.87 & 0 & 0 \\
\hline 18 & A. sinensis (Oliv.) Diels & succinic acid & $110-15-6$ & 118.1 & 29.62 & -0.44 & 0.01 & -0.41 & 2 & 4 \\
\hline 19 & A. sinensis (Oliv.) Diels & sitogluside & $474-58-8$ & 576.95 & 20.63 & -0.14 & 0.62 & 6.34 & 4 & 6 \\
\hline 20 & A. sinensis (Oliv.) Diels & beta-sitosterol & $83-46-5$ & 414.79 & 36.91 & 1.32 & 0.75 & 8.08 & 1 & 1 \\
\hline 21 & A. sinensis (Oliv.) Diels & 3-(4-hydroxy-3-methoxy-phenyl) acrylic acid & $537-98-4$ & 194.2 & 39.56 & 0.47 & 0.06 & 1.62 & 2 & 4 \\
\hline 22 & A. sinensis (Oliv.) Diels & D-galacturonic acid & $11100-11-1$ & 194.16 & 29.75 & -2 & 0.04 & -2.47 & 5 & 7 \\
\hline 23 & A. sinensis (Oliv.) Diels & (Z)-ferulic acid & 1014-83-1 & 194.2 & 54.97 & 0.53 & 0.06 & 1.62 & 2 & 4 \\
\hline
\end{tabular}




\begin{tabular}{|c|c|c|c|c|c|c|c|c|c|c|}
\hline 24 & A. sinensis (Oliv.) Diels & nicotinic acid & $123574-58-3$ & 123.12 & 47.65 & 0.34 & 0.02 & 0.28 & 1 & 3 \\
\hline 25 & A. sinensis (Oliv.) Diels & stigmasterol & $83-48-7$ & 412.77 & 43.83 & 1.44 & 0.76 & 7.64 & 1 & 1 \\
\hline 26 & A. sinensis (Oliv.) Diels & eucarvone & $503-93-5$ & 150.24 & 53.14 & 1.35 & 0.03 & 2.07 & 0 & 1 \\
\hline 27 & A. sinensis (Oliv.) Diels & farnesene & $18452-58-9$ & 204.39 & 17.42 & 1.95 & 0.05 & 5.52 & 0 & 0 \\
\hline 28 & A. sinensis (Oliv.) Diels & $\begin{array}{l}\text { (1S,4aR,8aR)-1-isopropyl-7-methyl-4-methylene-2 } \\
\text {,3,4a,5,6,8a-hexahydro-1H-naphthalene }\end{array}$ & $1460-97-5$ & 204.39 & 19.8 & 1.86 & 0.08 & 4.8 & 0 & 0 \\
\hline 29 & A. sinensis (Oliv.) Diels & vanillin & $121-33-5$ & 152.16 & 52 & 0.68 & 0.03 & 1.31 & 1 & 3 \\
\hline 30 & A. sinensis (Oliv.) Diels & heptanal & $111-71-7$ & 114.21 & 19.59 & 1.29 & 0.01 & 2.31 & 0 & 1 \\
\hline 31 & A. sinensis (Oliv.) Diels & dodekan & $112-40-3$ & 170.38 & 17.74 & 1.79 & 0.02 & 5.85 & 0 & 0 \\
\hline 32 & A. sinensis (Oliv.) Diels & bergamotene & $15438-93-4$ & 204.39 & 28.51 & 1.86 & 0.09 & 4.75 & 0 & 0 \\
\hline 33 & A. sinensis (Oliv.) Diels & cuminal & $122-03-2$ & 148.22 & 38.29 & 1.39 & 0.03 & 2.78 & 0 & 1 \\
\hline 34 & A. sinensis (Oliv.) Diels & loxanol V & $63393-82-8$ & 214.44 & 14.19 & 1.27 & 0.05 & 5.53 & 1 & 1 \\
\hline 35 & A. sinensis (Oliv.) Diels & tridecylene & $2437-56-1$ & 182.39 & 17.69 & 1.83 & 0.03 & 5.91 & 0 & 0 \\
\hline 36 & A. sinensis (Oliv.) Diels & $\begin{array}{l}(1 \mathrm{R}, 5 \mathrm{R})-2,7,7 \text {-trimethylbicyclo[3.1.1]hept-2-en-4- } \\
\text { one }\end{array}$ & $80-57-9$ & 150.24 & 50.63 & 1.27 & 0.06 & 1.94 & 0 & 1 \\
\hline 37 & A. sinensis (Oliv.) Diels & decanedioic acid & $32734-00-2$ & 202.28 & 16.23 & -0.01 & 0.05 & 2.33 & 2 & 4 \\
\hline 38 & A. sinensis (Oliv.) Diels & o-acetyl-p-cresol & $1450-72-2$ & 150.19 & 24.96 & 1.02 & 0.03 & 1.79 & 1 & 2 \\
\hline 39 & A. sinensis (Oliv.) Diels & nonanedioic acid & $32733-99-6$ & 188.25 & 16.9 & -0.04 & 0.04 & 1.87 & 2 & 4 \\
\hline 40 & A. sinensis (Oliv.) Diels & $(+)-$ ledol & $577-27-5$ & 222.41 & 16.96 & 1.43 & 0.12 & 3.2 & 1 & 1 \\
\hline 41 & A. sinensis (Oliv.) Diels & hypnon & $98-86-2$ & 120.16 & 48.19 & 1.36 & 0.02 & 1.57 & 0 & 1 \\
\hline 42 & A. sinensis (Oliv.) Diels & decanoic acid & $334-48-5$ & 172.3 & 26.74 & 0.96 & 0.03 & 3.63 & 1 & 2 \\
\hline 43 & A. sinensis (Oliv.) Diels & adenine & $73-24-5$ & 135.15 & 62.81 & -0.3 & 0.03 & -0.58 & 3 & 4 \\
\hline 44 & A. sinensis (Oliv.) Diels & $(+)$-cuparene & $16982-00-6$ & 202.37 & 38.26 & 1.88 & 0.07 & 4.72 & 0 & 0 \\
\hline 45 & A. sinensis (Oliv.) Diels & cis-thujopsene & $470-40-6$ & 204.39 & 56.43 & 1.84 & 0.12 & 4.08 & 0 & 0 \\
\hline 46 & A. sinensis (Oliv.) Diels & 3-butylidene-7-hydroxyphthalide & $93236-67-0$ & 204.24 & 62.68 & 1 & 0.08 & 2.74 & 1 & 3 \\
\hline 47 & A. sinensis (Oliv.) Diels & levistolid A & $88182-33-6$ & 380.52 & 2.15 & 0.94 & 0.82 & 4.97 & 0 & 4 \\
\hline 48 & A. sinensis (Oliv.) Diels & allocymene & $673-84-7$ & 136.26 & 14.89 & 1.85 & 0.02 & 3.58 & 0 & 0 \\
\hline 49 & A. sinensis (Oliv.) Diels & 3-butylidene phthalide & $551-08-6$ & 188.24 & 42.44 & 1.32 & 0.07 & 3 & 0 & 2 \\
\hline
\end{tabular}




\begin{tabular}{|c|c|c|c|c|c|c|c|c|c|c|}
\hline 50 & A. sinensis (Oliv.) Diels & senkyunolide $\mathrm{C}$ & $63038-10-8$ & 204.24 & 46.8 & 0.87 & 0.08 & 2.74 & 1 & 3 \\
\hline 51 & A. sinensis (Oliv.) Diels & senkyunolide D & $93236-67-0$ & 222.26 & 79.13 & 0.12 & 0.1 & 1.8 & 1 & 4 \\
\hline 52 & A. sinensis (Oliv.) Diels & senkyunolide E & $91652-78-7$ & 204.24 & 34.4 & 0.55 & 0.08 & 1.9 & 1 & 3 \\
\hline 53 & A. sinensis (Oliv.) Diels & 4-octanone & $589-63-9$ & 128.24 & 19.37 & 1.37 & 0.01 & 2.46 & 0 & 1 \\
\hline 54 & A. sinensis (Oliv.) Diels & (6R)-6-butylcyclohepta-1,4-diene & $33156-91-1$ & 150.29 & 31.69 & 1.85 & 0.02 & 3.93 & 0 & 0 \\
\hline 55 & A. sinensis (Oliv.) Diels & cis-ligustilide & $4431-01-0$ & 190.26 & 51.3 & 1.3 & 0.07 & 2.94 & 0 & 2 \\
\hline 56 & A. sinensis (Oliv.) Diels & methylbutenol & $115-18-4$ & 86.15 & 54.58 & 1.12 & 0.01 & 0.84 & 1 & 1 \\
\hline 57 & A. sinensis (Oliv.) Diels & tris(4-methylphenoxy) aluminum & $72269-62-6$ & 108.15 & 51.99 & 1.56 & 0.01 & 2.05 & 1 & 1 \\
\hline 58 & A. sinensis (Oliv.) Diels & $\begin{array}{l}\text { (4S)-1-methyl-4-(6-methylhepta-1,5-dien-2-yl)cycl } \\
\text { ohexene }\end{array}$ & $495-61-4$ & 204.39 & 20.3 & 1.89 & 0.06 & 5.33 & 0 & 0 \\
\hline 59 & A. sinensis (Oliv.) Diels & guasol & $8021-39-4$ & 124.15 & 51.6 & 1.28 & 0.02 & 1.55 & 1 & 2 \\
\hline 60 & A. sinensis (Oliv.) Diels & liquified Phenol & $108-95-2$ & 94.12 & 36.05 & 1.5 & 0.01 & 1.56 & 1 & 1 \\
\hline 61 & A. sinensis (Oliv.) Diels & cadinene & $523-47-7$ & 204.39 & 17.12 & 1.88 & 0.08 & 4.75 & 0 & 0 \\
\hline 62 & A. sinensis (Oliv.) Diels & acoradiene & $24048-44-0$ & 204.39 & 36.73 & 1.85 & 0.07 & 4.75 & 0 & 0 \\
\hline 63 & A. sinensis (Oliv.) Diels & maruzen $\mathrm{M}$ & $29471-88-3$ & 122.18 & 48.44 & 1.57 & 0.02 & 2.51 & 1 & 1 \\
\hline 64 & A. sinensis (Oliv.) Diels & o-cresol & $95-48-7$ & 108.15 & 62.45 & 1.57 & 0.02 & 2.05 & 1 & 1 \\
\hline 65 & A. sinensis (Oliv.) Diels & riligustilide & $89354-45-0$ & 380.52 & 9.83 & 0.79 & 0.7 & 5.17 & 0 & 4 \\
\hline 66 & A. sinensis (Oliv.) Diels & beta-terpinene & $99-84-3$ & 136.26 & 42.29 & 1.85 & 0.02 & 3.5 & 0 & 0 \\
\hline 67 & A. sinensis (Oliv.) Diels & butyraldehyde & $123-72-8$ & 72.12 & 68.66 & 1.18 & 0 & 0.94 & 0 & 1 \\
\hline 68 & A. sinensis (Oliv.) Diels & ethol & $36311-34-9$ & 242.5 & 13.32 & 1.31 & 0.08 & 6.45 & 1 & 1 \\
\hline 69 & A. sinensis (Oliv.) Diels & 4-methoxybenzoic acid & $100-09-4$ & 152.16 & 29.69 & 0.69 & 0.03 & 1.42 & 1 & 3 \\
\hline 70 & A. sinensis (Oliv.) Diels & (2S)-2-amino-4-methylsulfanylbutanoic acid & $26062-47-5$ & 149.24 & 70.87 & 0.06 & 0.01 & -0.27 & 3 & 3 \\
\hline 71 & A. sinensis (Oliv.) Diels & 3,4-dimethylbenzaldehyde & $5973-71-7$ & 134.19 & 39.99 & 1.4 & 0.02 & 2.56 & 0 & 1 \\
\hline 72 & A. sinensis (Oliv.) Diels & ethylbenzaldehyde & $4748-78-1$ & 134.19 & 40.95 & 1.4 & 0.02 & 2.53 & 0 & 1 \\
\hline 73 & A. sinensis (Oliv.) Diels & tmhydrop & $700-13-0$ & 152.21 & 54.42 & 1.21 & 0.03 & 2.75 & 2 & 2 \\
\hline 74 & A. sinensis (Oliv.) Diels & bicycloelemene & $32531-56-9$ & 204.39 & 20.89 & 1.88 & 0.08 & 4.89 & 0 & 0 \\
\hline 75 & A. sinensis (Oliv.) Diels & 1-(2,4-dihydroxyphenyl)ethanone & $89-84-9$ & 152.16 & 36.49 & 0.67 & 0.03 & 1.03 & 2 & 3 \\
\hline 76 & A. sinensis (Oliv.) Diels & phthalic acid & $88-99-3$ & 166.14 & 17.74 & -0.05 & 0.04 & 1.04 & 2 & 4 \\
\hline
\end{tabular}




\begin{tabular}{|c|c|c|c|c|c|c|c|c|c|c|}
\hline 77 & A. sinensis (Oliv.) Diels & $\begin{array}{l}\text { (1R,2S,4R)-1-ethyl-1-methyl-2,4-bis(1-methylethy } \\
\text { 1)cyclohexane }\end{array}$ & $515-12-8$ & 210.45 & 15.01 & 1.8 & 0.06 & 5.57 & 0 & 0 \\
\hline 78 & A. sinensis (Oliv.) Diels & alkenes & $68526-58-9$ & 168.36 & 17.74 & 1.8 & 0.02 & 5.46 & 0 & 0 \\
\hline 79 & A. sinensis (Oliv.) Diels & phosphatdic acid & $97281-52-2$ & 228.11 & 19.32 & -1.22 & 0.05 & -1.55 & 2 & 8 \\
\hline 80 & A. sinensis (Oliv.) Diels & L-beta,gamma-dimyristoyl-alpha-cephalin & $90989-93-8$ & 635.97 & 20.69 & -0.43 & 0.47 & 9.49 & 3 & 9 \\
\hline 81 & A. sinensis (Oliv.) Diels & phosphatidylinositol & $383907-36-6$ & 390.27 & 4.63 & -2.34 & 0.29 & -3.85 & 6 & 13 \\
\hline 82 & A. sinensis (Oliv.) Diels & phosphatidylinositol_qt & $383907-36-6$ & 228.11 & 12.66 & -1.15 & 0.05 & -1.55 & 2 & 8 \\
\hline 83 & A. sinensis (Oliv.) Diels & 2-benzofuran-1,3-dione & $85-44-9$ & 148.12 & 47.31 & 0.56 & 0.04 & 1.3 & 0 & 3 \\
\hline 84 & A. sinensis (Oliv.) Diels & sedanolide & $6415-59-4$ & 194.3 & 62.46 & 1.24 & 0.07 & 3.37 & 0 & 2 \\
\hline 85 & A. sinensis (Oliv.) Diels & senkyunolide & $63038-10-8$ & 192.28 & 68.28 & 1.28 & 0.07 & 3.19 & 0 & 2 \\
\hline 86 & A. sinensis (Oliv.) Diels & sphingomyelin & $85187-10-6$ & 493.73 & 0.31 & -0.46 & 0.51 & 3.18 & 3 & 7 \\
\hline 87 & A. sinensis (Oliv.) Diels & Isotetandrine & $477-57-6$ & 622.82 & 10.42 & 0.95 & 0.1 & 7.22 & 0 & 8 \\
\hline 88 & A. sinensis (Oliv.) Diels & $\alpha$-acoradiene & $28400-13-7$ & 204.39 & 40.98 & 1.82 & 0.07 & 4.51 & 0 & 0 \\
\hline 89 & A. sinensis (Oliv.) Diels & (-)-cedrene & $469-61-4$ & 204.39 & 55.56 & 1.79 & 0.1 & 4.12 & 0 & 0 \\
\hline 90 & A. sinensis (Oliv.) Diels & $\alpha$-copaene & $3856-25-5$ & 204.39 & 29.33 & 1.83 & 0.12 & 4.17 & 0 & 0 \\
\hline 91 & A. sinensis (Oliv.) Diels & $\begin{array}{l}\text { (1R,4R,5S)-4-isopropenyl-1,8-dimethylspiro[4.5]d } \\
\text { ec-8-ene }\end{array}$ & N/A & 204.39 & 40.65 & 1.83 & 0.07 & 4.75 & 0 & 0 \\
\hline 92 & A. sinensis (Oliv.) Diels & 2,6-di(phenyl)thiopyran-4-thione & $1029-97-6$ & 280.43 & 69.13 & 1.74 & 0.15 & 4.62 & 0 & 0 \\
\hline 93 & A. sinensis (Oliv.) Diels & o-xylenol & $526-75-0$ & 122.18 & 53.13 & 1.62 & 0.02 & 2.54 & 1 & 1 \\
\hline 94 & A. sinensis (Oliv.) Diels & 2,4,6-trimethyl-Octane & $62016-37-9$ & 156.35 & 29.14 & 1.81 & 0.02 & 4.78 & 0 & 0 \\
\hline 95 & A. sinensis (Oliv.) Diels & mesitaldehyde & $487-68-3$ & 148.22 & 37.8 & 1.54 & 0.03 & 3.05 & 0 & 1 \\
\hline 96 & A. sinensis (Oliv.) Diels & isoxylaldehyde & $5779-94-2$ & 134.19 & 38.85 & 1.39 & 0.02 & 2.56 & 0 & 1 \\
\hline 97 & A. sinensis (Oliv.) Diels & (E)-octadec-3-ene & $7206-19-1$ & 252.54 & 19.5 & 1.86 & 0.09 & 8.14 & 0 & 0 \\
\hline 98 & A. sinensis (Oliv.) Diels & 2-valerylbenzoic acid & $64624-87-9$ & 206.26 & 78.26 & 0.61 & 0.06 & 2.75 & 1 & 3 \\
\hline 99 & A. sinensis (Oliv.) Diels & (Z)-2-hexenyl hexanoate & $53398-86-0$ & 198.34 & 19.39 & 1.26 & 0.04 & 3.99 & 0 & 2 \\
\hline 100 & A. sinensis (Oliv.) Diels & 2-methylhexadecanoic acid & $2490-53-1$ & 270.51 & 20.23 & 1.07 & 0.11 & 6.83 & 1 & 2 \\
\hline 101 & A. sinensis (Oliv.) Diels & cis-isoeugenol & $5912-86-7$ & 164.22 & 20.73 & 1.43 & 0.04 & 2.5 & 1 & 2 \\
\hline 102 & A. sinensis (Oliv.) Diels & 2-methyl-5-decanone & $54410-89-8$ & 310.63 & 20.4 & 1.42 & 0.11 & 7.99 & 0 & 1 \\
\hline
\end{tabular}




\begin{tabular}{|c|c|c|c|c|c|c|c|c|c|c|}
\hline 103 & A. sinensis (Oliv.) Diels & 2-methyldodecan-5-one & $\mathrm{N} / \mathrm{A}$ & 198.39 & 13.16 & 1.43 & 0.04 & 4.54 & 0 & 1 \\
\hline 104 & A. sinensis (Oliv.) Diels & 3,7-dimethyl-nonane & $17302-32-8$ & 156.35 & 16.97 & 1.78 & 0.02 & 4.98 & 0 & 0 \\
\hline 105 & A. sinensis (Oliv.) Diels & $\begin{array}{l}\text { 1,1,5-trimethyl-2-formylcyclohexa-2,5-diene-4-on } \\
\mathrm{e}\end{array}$ & $23985-22-0$ & 164.22 & 48.94 & 0.82 & 0.04 & 1.64 & 0 & 2 \\
\hline 106 & A. sinensis (Oliv.) Diels & 4-methyl-6-hepten-3-one & 26118-97-8 & 126.22 & 78.38 & 1.39 & 0.01 & 2.07 & 0 & 1 \\
\hline 107 & A. sinensis (Oliv.) Diels & 6-ethylresorcinol & $2896-60-8$ & 138.18 & 46.45 & 1.13 & 0.03 & 2.24 & 2 & 2 \\
\hline 108 & A. sinensis (Oliv.) Diels & 5-indolol & $1953-54-4$ & 133.16 & 63.14 & 1.38 & 0.03 & 1.86 & 2 & 1 \\
\hline 109 & A. sinensis (Oliv.) Diels & undecanol-6-ol & $23708-56-7$ & 172.35 & 25.77 & 1.19 & 0.02 & 4.15 & 1 & 1 \\
\hline 110 & A. sinensis (Oliv.) Diels & 7,10-pentadecadiynoic acid & 22117-06-2 & 234.37 & 41.5 & 1.32 & 0.09 & 5.27 & 1 & 2 \\
\hline 111 & A. sinensis (Oliv.) Diels & $\begin{array}{l}\text { 4-chloro-N-[1-methyl-5-[[1-methyl-5-[[1-methyl-5 } \\
\text {-(2-morpholinoethylcarbamoyl)pyrrol-3-yl]carbam } \\
\text { oyl]pyrrol-3-yl]carbamoyl]pyrrol-3-yl]-5-[2-(2-pyr } \\
\text { idyl)ethylamino]isothiazole-3-carboxamide }\end{array}$ & $210045-96-8$ & 762.38 & 7.18 & -0.21 & 0.31 & 2.93 & 5 & 13 \\
\hline 112 & A. sinensis (Oliv.) Diels & amyl ketone & $71808-49-6$ & 170.33 & 18.65 & 1.31 & 0.02 & 3.83 & 0 & 1 \\
\hline 113 & A. sinensis (Oliv.) Diels & isoamylbenzene & 2049-94-7 & 148.27 & 35.69 & 1.84 & 0.03 & 3.94 & 0 & 0 \\
\hline 114 & A. sinensis (Oliv.) Diels & $\begin{array}{l}\text { (Z)-2-[[(Z)-2-methylbut-2-enoyl]oxymethyl]but-2- } \\
\text { enoic acid }\end{array}$ & $69188-40-5$ & 198.24 & 77.1 & 0.49 & 0.04 & 2.15 & 1 & 4 \\
\hline 115 & A. sinensis (Oliv.) Diels & tropone & $539-80-0$ & 106.13 & 47.41 & 1.25 & 0.01 & 1.14 & 0 & 1 \\
\hline 116 & A. sinensis (Oliv.) Diels & aromadendrene & $489-39-4$ & 204.39 & 18.21 & 1.83 & 0.1 & 4.22 & 0 & 0 \\
\hline 117 & A. sinensis (Oliv.) Diels & butanoic acid & $107-92-6$ & 88.12 & 21.62 & 0.69 & 0 & 0.89 & 1 & 2 \\
\hline 118 & A. sinensis (Oliv.) Diels & (3S)-3-butyl-3H-isobenzofuran-1-one & $6066-49-5$ & 190.26 & 55.05 & 1.3 & 0.07 & 3.25 & 0 & 2 \\
\hline 119 & A. sinensis (Oliv.) Diels & (trans)-camphoric acid & $6066-49-5$ & 200.26 & 99.13 & 0.1 & 0.07 & 1.48 & 2 & 4 \\
\hline 120 & A. sinensis (Oliv.) Diels & (3E)-3-butylidene-7-hydroxy-2-benzofuran-1-one & $103659-69-4$ & 204.24 & 42.17 & 1.03 & 0.08 & 2.74 & 1 & 3 \\
\hline 121 & A. sinensis (Oliv.) Diels & coniferyl ferulate & $63644-62-2$ & 356.4 & 4.54 & 0.71 & 0.39 & 3.64 & 2 & 6 \\
\hline 122 & A. sinensis (Oliv.) Diels & lecithin & $8002-43-5$ & 678.06 & 0.31 & 0.16 & 0.4 & 8.77 & 0 & 8 \\
\hline 123 & A. sinensis (Oliv.) Diels & 2,4-xylylaldehyde & $15764-16-6$ & 134.19 & 39.33 & 1.42 & 0.03 & 2.56 & 0 & 1 \\
\hline 124 & A. sinensis (Oliv.) Diels & m-ethylphenol & $620-17-7$ & 122.18 & 51.3 & 1.55 & 0.02 & 2.51 & 1 & 1 \\
\hline 125 & A. sinensis (Oliv.) Diels & 1,5,5,6-tetramethyl-1,3-Cyclohexadiene & $514-94-3$ & 136.26 & 39.22 & 1.82 & 0.03 & 3 & 0 & 0 \\
\hline
\end{tabular}




\begin{tabular}{|c|c|c|c|c|c|c|c|c|c|c|}
\hline 126 & L. chuanxiong Hort & D-camphene & $5796-03-6$ & 136.26 & 34.98 & 1.81 & 0.04 & 2.93 & 0 & 0 \\
\hline 127 & L. chuanxiong Hort & alpha-humulene & $19132-75-3$ & 204.39 & 22.98 & 1.88 & 0.06 & 5.04 & 0 & 0 \\
\hline 128 & L. chuanxiong Hort & alpha-curcumene & $4176-17-4$ & 202.37 & 4.68 & 1.93 & 0.06 & 5.34 & 0 & 0 \\
\hline 129 & L. chuanxiong Hort & $\begin{array}{l}\text { 2-[(1R,3S,4S)-3-isopropenyl-4-methyl-4-vinylcycl } \\
\text { ohexyl]propan-2-ol }\end{array}$ & $639-99-6$ & 222.41 & 19.03 & 1.37 & 0.07 & 3.7 & 1 & 1 \\
\hline 130 & L. chuanxiong Hort & beta-Selinene & $17066-67-0$ & 204.39 & 24.39 & 1.83 & 0.08 & 4.81 & 0 & 0 \\
\hline 131 & L. chuanxiong Hort & palmitic acid & $67701-02-4$ & 256.48 & 19.3 & 1.09 & 0.1 & 6.37 & 1 & 2 \\
\hline 132 & L. chuanxiong Hort & 4-hydroxybenzenecarboxylic acid & $99-96-7$ & 138.13 & 30.15 & 0.39 & 0.03 & 1.17 & 2 & 3 \\
\hline 133 & L. chuanxiong Hort & vanillic acid & $121-34-6$ & 168.16 & 35.47 & 0.43 & 0.04 & 1.15 & 2 & 4 \\
\hline 134 & L. chuanxiong Hort & nonanal & $75718-12-6$ & 142.27 & 40.28 & 1.31 & 0.02 & 3.22 & 0 & 1 \\
\hline 135 & L. chuanxiong Hort & cymol & $4939-75-7$ & 134.24 & 27.2 & 1.86 & 0.02 & 3.51 & 0 & 0 \\
\hline 136 & L. chuanxiong Hort & (L)-alpha-terpineol & $10482-56-1$ & 154.28 & 48.8 & 1.39 & 0.03 & 2.42 & 1 & 1 \\
\hline 137 & L. chuanxiong Hort & 1,8 -cineole & $470-82-6$ & 154.28 & 39.73 & 1.57 & 0.05 & 2.15 & 0 & 1 \\
\hline 138 & L. chuanxiong Hort & (-)-alpha-pinene & $7785-26-4$ & 136.26 & 46.25 & 1.85 & 0.05 & 2.87 & 0 & 0 \\
\hline 139 & L. chuanxiong Hort & (1S,5S)-6,6-dimethyl-2-methylene-norpinane & $18172-67-3$ & 136.26 & 44.84 & 1.8 & 0.05 & 2.93 & 0 & 0 \\
\hline 140 & L. chuanxiong Hort & (9Z,12Z)-octadeca-9,12-dienoic acid & $2197-37-7$ & 280.5 & 41.9 & 1.16 & 0.14 & 6.39 & 1 & 2 \\
\hline 141 & L. chuanxiong Hort & $\begin{array}{l}\text { 2-[(2S,5S,6S)-6,10-dimethylspiro[4.5]dec-9-en-2-y } \\
\text { 1]propan-2-ol }\end{array}$ & $1460-73-7$ & 222.41 & 37.62 & 1.44 & 0.09 & 3.67 & 1 & 1 \\
\hline 142 & L. chuanxiong Hort & furol & $98-01-1$ & 96.09 & 34.35 & 1.08 & 0.01 & 0.99 & 0 & 2 \\
\hline 143 & L. chuanxiong Hort & L-bornyl acetate & $6626-35-3$ & 196.32 & 65.52 & 1.29 & 0.08 & 2.35 & 0 & 2 \\
\hline 144 & L. chuanxiong Hort & myrcene & $123-35-3$ & 136.26 & 24.96 & 1.84 & 0.02 & 3.69 & 0 & 0 \\
\hline 145 & L. chuanxiong Hort & (R)-linalool & $126-91-0$ & 154.28 & 39.8 & 1.33 & 0.02 & 2.74 & 1 & 1 \\
\hline 146 & L. chuanxiong Hort & (S)-(+)-alpha-Phellandrene & $2243-33-6$ & 136.26 & 27.9 & 1.87 & 0.02 & 3.25 & 0 & 0 \\
\hline 147 & L. chuanxiong Hort & p-ocimene & $2243-33-6$ & 136.26 & 15.06 & 1.85 & 0.02 & 3.63 & 0 & 0 \\
\hline 148 & L. chuanxiong Hort & moslene & $99-85-4$ & 136.26 & 33.02 & 1.88 & 0.02 & 3.45 & 0 & 0 \\
\hline 149 & L. chuanxiong Hort & $\begin{array}{l}(1 \mathrm{~S}, 2 \mathrm{R}, 4 \mathrm{R}) \text {-2,4-diisopropenyl-1-methyl-1-vinylcyc } \\
\text { lohexane }\end{array}$ & $515-13-9$ & 204.39 & 28.62 & 1.85 & 0.06 & 4.79 & 0 & 0 \\
\hline 150 & L. chuanxiong Hort & methyleugenol & $6380-24-1$ & 178.25 & 73.36 & 1.47 & 0.04 & 2.81 & 0 & 2 \\
\hline
\end{tabular}




\begin{tabular}{|c|c|c|c|c|c|c|c|c|c|c|}
\hline 151 & L. chuanxiong Hort & aromadendrene & $489-39-4$ & 204.39 & 55.74 & 1.81 & 0.1 & 4.22 & 0 & 0 \\
\hline 152 & L. chuanxiong Hort & caffeic acid & $71693-97-5$ & 180.17 & 25.76 & 0.21 & 0.05 & 1.37 & 3 & 4 \\
\hline 153 & L. chuanxiong Hort & L-limonen & $5989-54-8$ & 136.26 & 38.09 & 1.83 & 0.02 & 3.5 & 0 & 0 \\
\hline 154 & L. chuanxiong Hort & tereben & $69073-38-7$ & 136.26 & 29.62 & 1.86 & 0.02 & 3.64 & 0 & 0 \\
\hline 155 & L. chuanxiong Hort & $\begin{array}{l}\text { (1S,5S)-1-isopropyl-4-methylenebicyclo[3.1.0]hex } \\
\text { ane }\end{array}$ & $87-41-5$ & 136.26 & 46.21 & 1.83 & 0.04 & 2.93 & 0 & 0 \\
\hline 156 & L. chuanxiong Hort & $(+)$-delta(3)-carene & $498-15-7$ & 136.26 & 45.2 & 1.84 & 0.04 & 2.87 & 0 & 0 \\
\hline 157 & L. chuanxiong Hort & undekansaeure & $112-37-8$ & 186.33 & 30.14 & 0.98 & 0.03 & 4.09 & 1 & 2 \\
\hline 158 & L. chuanxiong Hort & sitogluside & $474-58-8$ & 576.95 & 20.63 & -0.14 & 0.62 & 6.34 & 4 & 6 \\
\hline 159 & L. chuanxiong Hort & sitosterol & $83-46-5$ & 414.79 & 36.91 & 1.32 & 0.75 & 8.08 & 1 & 1 \\
\hline 160 & L. chuanxiong Hort & folsaeure & $33609-88-0$ & 441.45 & 68.96 & -1.5 & 0.71 & 0.01 & 7 & 13 \\
\hline 161 & L. chuanxiong Hort & (S)-p-menth-1-en-4-ol & $2438-10-0$ & 154.28 & 81.41 & 1.36 & 0.03 & 2.55 & 1 & 1 \\
\hline 162 & L. chuanxiong Hort & vanillin & $121-33-5$ & 152.16 & 52 & 0.68 & 0.03 & 1.31 & 1 & 3 \\
\hline 163 & L. chuanxiong Hort & hexanal & $66-25-1$ & 100.18 & 55.71 & 1.25 & 0.01 & 1.85 & 0 & 1 \\
\hline 164 & L. chuanxiong Hort & pentylfuran & $64079-01-2$ & 138.23 & 54.59 & 1.72 & 0.02 & 3.12 & 0 & 1 \\
\hline 165 & L. chuanxiong Hort & oleic acid & $17156-84-2$ & 282.52 & 33.13 & 1.17 & 0.14 & 6.84 & 1 & 2 \\
\hline 166 & L. chuanxiong Hort & (R)-(-)-alpha-phellandrene & $4221-98-1$ & 136.26 & 27.51 & 1.86 & 0.02 & 3.25 & 0 & 0 \\
\hline 167 & L. chuanxiong Hort & heptanal & $111-71-7$ & 114.21 & 19.59 & 1.29 & 0.01 & 2.31 & 0 & 1 \\
\hline 168 & L. chuanxiong Hort & caprylaldehyde & $124-13-0$ & 128.24 & 19.07 & 1.3 & 0.01 & 2.77 & 0 & 1 \\
\hline 169 & L. chuanxiong Hort & o-cymol & $1329-98-2$ & 134.24 & 51.89 & 1.88 & 0.02 & 3.51 & 0 & 0 \\
\hline 170 & L. chuanxiong Hort & sucrose & $25702-74-3$ & 342.34 & 7.17 & -2.89 & 0.23 & -4.31 & 8 & 11 \\
\hline 171 & L. chuanxiong Hort & stearic acid & 609343-71-7 & 284.54 & 17.83 & 1.15 & 0.14 & 7.28 & 1 & 2 \\
\hline 172 & L. chuanxiong Hort & n-pentadecane & $629-62-9$ & 212.47 & 13.98 & 1.81 & 0.05 & 7.22 & 0 & 0 \\
\hline 173 & L. chuanxiong Hort & methyl palmitate & $112-39-0$ & 270.51 & 18.09 & 1.37 & 0.12 & 6.62 & 0 & 2 \\
\hline 174 & L. chuanxiong Hort & tetradecane & $90622-46-1$ & 198.44 & 15.94 & 1.79 & 0.04 & 6.76 & 0 & 0 \\
\hline 175 & L. chuanxiong Hort & beta-elemene & $122-78-1$ & 204.39 & 25.63 & 1.84 & 0.06 & 4.79 & 0 & 0 \\
\hline 176 & L. chuanxiong Hort & terpilene & $99-86-5$ & 136.26 & 33.95 & 1.84 & 0.02 & 3.45 & 0 & 0 \\
\hline 177 & L. chuanxiong Hort & (R)-p-menth-1-en-4-ol & $20126-76-5$ & 154.28 & 32.16 & 1.33 & 0.03 & 2.55 & 1 & 1 \\
\hline
\end{tabular}




\begin{tabular}{|c|c|c|c|c|c|c|c|c|c|c|}
\hline 178 & L. chuanxiong Hort & alpha-Farnesene & $502-61-4$ & 204.39 & 21.7 & 1.97 & 0.05 & 5.46 & 0 & 0 \\
\hline 179 & L. chuanxiong Hort & $\begin{array}{l}\text { (4E)-1-methyl-4-(6-methylhept-5-en-2-ylidene)cyc } \\
\text { lohexene }\end{array}$ & $495-62-5$ & 204.39 & 20.78 & 1.94 & 0.06 & 5.47 & 0 & 0 \\
\hline 180 & L. chuanxiong Hort & isoledene & $95910-36-4$ & 204.39 & 49.01 & 1.82 & 0.1 & 4.36 & 0 & 0 \\
\hline 181 & L. chuanxiong Hort & ethylpalmitate & $628-97-7$ & 284.54 & 18.99 & 1.41 & 0.14 & 6.97 & 0 & 2 \\
\hline 182 & L. chuanxiong Hort & 5-isopropyl-2-methylbicyclo[3.1.0]hex-2-ene & $1406-51-5$ & 136.26 & 47.19 & 1.82 & 0.04 & 2.87 & 0 & 0 \\
\hline 183 & L. chuanxiong Hort & $\begin{array}{l}\text { (1R,5R,7S)-4,7-dimethyl-7-(4-methylpent-3-enyl) } \\
\text { bicyclo[3.1.1]hept-3-ene }\end{array}$ & $79-92-5$ & 204.39 & 16.23 & 1.86 & 0.09 & 4.7 & 0 & 0 \\
\hline 184 & L. chuanxiong Hort & (4S)-4-isopropylcyclohexene-1-carbaldehyde & 21391-98-0 & 152.26 & 40.36 & 1.36 & 0.03 & 2.86 & 0 & 1 \\
\hline 185 & L. chuanxiong Hort & (S)-2,2,3-trimethylcyclopent-3-ene-1-acetaldehyde & $23727-15-3$ & 152.26 & 45.18 & 1.32 & 0.03 & 2.19 & 0 & 1 \\
\hline 186 & L. chuanxiong Hort & o-acetyl-p-cresol & $1450-72-2$ & 150.19 & 24.96 & 1.02 & 0.03 & 1.79 & 1 & 2 \\
\hline 187 & L. chuanxiong Hort & phenylmethanol & $1336-27-2$ & 108.15 & 58.68 & 1.08 & 0.01 & 1.23 & 1 & 1 \\
\hline 188 & L. chuanxiong Hort & (-)-globulol & $489-41-8$ & 222.41 & 85.51 & 1.29 & 0.12 & 3.2 & 1 & 1 \\
\hline 189 & L. chuanxiong Hort & mandenol & $544-35-4$ & 308.56 & 42 & 1.46 & 0.19 & 6.99 & 0 & 2 \\
\hline 190 & L. chuanxiong Hort & germacrene & $23986-74-5$ & 208.43 & 15.06 & 1.82 & 0.06 & 5.58 & 0 & 0 \\
\hline 191 & L. chuanxiong Hort & methyl linoleate & $112-63-0$ & 294.53 & 41.93 & 1.44 & 0.17 & 6.64 & 0 & 2 \\
\hline 192 & L. chuanxiong Hort & crysophanol & $481-74-3$ & 254.25 & 18.64 & 0.62 & 0.21 & 2.76 & 2 & 4 \\
\hline 193 & L. chuanxiong Hort & uracil & $66-22-8$ & 112.1 & 42.53 & 0.05 & 0.02 & -1.01 & 2 & 4 \\
\hline 194 & L. chuanxiong Hort & 4-hydroxy-3-methoxystyrene & $7786-61-0$ & 150.19 & 38.39 & 1.36 & 0.03 & 2.1 & 1 & 2 \\
\hline 195 & L. chuanxiong Hort & polyriboadenosine & $30143-02-3$ & 267.28 & 15.98 & -1.56 & 0.18 & -2.02 & 5 & 8 \\
\hline 196 & L. chuanxiong Hort & adenine & $73-24-5$ & 135.15 & 62.81 & -0.3 & 0.03 & -0.58 & 3 & 4 \\
\hline 197 & L. chuanxiong Hort & methyl pentadecanoate & $7132-64-1$ & 256.48 & 18.82 & 1.37 & 0.1 & 6.16 & 0 & 2 \\
\hline 198 & L. chuanxiong Hort & (+)-beta-phellandrene & $555-10-2$ & 136.26 & 40.3 & 1.83 & 0.02 & 3.31 & 0 & 0 \\
\hline 199 & L. chuanxiong Hort & thymol & $89-83-8$ & 150.24 & 41.47 & 1.6 & 0.03 & 3.24 & 1 & 1 \\
\hline 200 & L. chuanxiong Hort & alpha-cubebene & $17699-14-8$ & 204.39 & 16.73 & 1.83 & 0.11 & 4.17 & 0 & 0 \\
\hline 201 & L. chuanxiong Hort & $(+)$-alpha-funebrene & 50894-66-1 & 204.39 & 52.87 & 1.79 & 0.1 & 4.12 & 0 & 0 \\
\hline 202 & L. chuanxiong Hort & gem-dimethylcyclopentane & $1638-26-2$ & 98.21 & 41.22 & 1.78 & 0.01 & 2.74 & 0 & 0 \\
\hline 203 & L. chuanxiong Hort & 3-butylidene-7-hydroxyphthalide & $93236-67-0$ & 204.24 & 62.68 & 1 & 0.08 & 2.74 & 1 & 3 \\
\hline
\end{tabular}




\begin{tabular}{|c|c|c|c|c|c|c|c|c|c|c|}
\hline 204 & L. chuanxiong Hort & senkyunolide $\mathrm{K}$ & $114569-33-4$ & 208.28 & 61.75 & 0.52 & 0.08 & 2.09 & 1 & 3 \\
\hline 205 & L. chuanxiong Hort & $\begin{array}{l}\text { (3Z,6S,7R)-3-butylidene-6-butyryl-7-hydroxy-4,5, } \\
\text { 6,7-tetrahydroisobenzofuran-1-one }\end{array}$ & $114569-34-5$ & 278.38 & 3.41 & 0.08 & 0.16 & 2.69 & 1 & 4 \\
\hline 206 & L. chuanxiong Hort & senkyunolide $\mathrm{N}$ & $140694-58-2$ & 226.3 & 37.27 & -0.15 & 0.1 & 1.57 & 2 & 4 \\
\hline 207 & L. chuanxiong Hort & levistolid A & $88182-33-6$ & 380.52 & 2.15 & 0.94 & 0.82 & 4.97 & 0 & 4 \\
\hline 208 & L. chuanxiong Hort & senkyunolide $\mathrm{P}$ & $142864-23-1$ & 382.54 & 9.38 & 0.92 & 0.81 & 5.22 & 0 & 4 \\
\hline 209 & L. chuanxiong Hort & senkyunolide Q & $142235-81-2$ & 278.38 & 26.84 & 0.42 & 0.16 & 2.69 & 1 & 4 \\
\hline 210 & L. chuanxiong Hort & senkyunolide R & $172549-37-0$ & 240.28 & 13.14 & -0.76 & 0.11 & 0.22 & 3 & 5 \\
\hline 211 & L. chuanxiong Hort & 1,1-diethoxybutane & $3658-95-5$ & 146.26 & 29.28 & 1.3 & 0.01 & 1.89 & 0 & 2 \\
\hline 212 & L. chuanxiong Hort & valerophenone & $1009-14-9$ & 162.25 & 42.58 & 1.46 & 0.03 & 3.15 & 0 & 1 \\
\hline 213 & L. chuanxiong Hort & $\begin{array}{l}\text { (1S,5S)-7,7-dimethyl-2-methylenebicyclo[3.1.1] he } \\
\text { pt-3-ene }\end{array}$ & $4080-46-0$ & 134.24 & 37.71 & 1.8 & 0.05 & 2.48 & 0 & 0 \\
\hline 214 & L. chuanxiong Hort & Z-6,8',7,3'-diligustilide & N/A & 380.52 & 11.98 & 0.74 & 0.7 & 5.17 & 0 & 4 \\
\hline 215 & L. chuanxiong Hort & allocymene & $673-84-7$ & 136.26 & 14.89 & 1.85 & 0.02 & 3.58 & 0 & 0 \\
\hline 216 & L. chuanxiong Hort & butylidenephthalide & $551-08-6$ & 188.24 & 42.44 & 1.32 & 0.07 & 3 & 0 & 2 \\
\hline 217 & L. chuanxiong Hort & alpha-selinene & $473-13-2$ & 218.42 & 31.81 & 1.82 & 0.1 & 4.96 & 0 & 0 \\
\hline 218 & L. chuanxiong Hort & $\begin{array}{l}\text { 1,2,3,4,4a,7-hexahydro-1,6-dimethyl-4-(1-methylet } \\
\text { hyl)-naphthalene }\end{array}$ & $\mathrm{N} / \mathrm{A}$ & 162.3 & 19.03 & 1.88 & 0.05 & 3.79 & 0 & 0 \\
\hline 219 & L. chuanxiong Hort & augustic-acid & $26707-60-8$ & 472.78 & 21.08 & 0.18 & 0.74 & 5.46 & 3 & 4 \\
\hline 220 & L. chuanxiong Hort & m-ethyltoluene & $620-14-4$ & 120.21 & 50.77 & 1.87 & 0.02 & 3.26 & 0 & 0 \\
\hline 221 & L. chuanxiong Hort & $\begin{array}{l}\text { (1R,4S,5R)-4-isopropenyl-1,8-dimethylspiro[4.5]d } \\
\text { ec-8-ene }\end{array}$ & $28400-13-7$ & 204.39 & 40.01 & 1.85 & 0.07 & 4.75 & 0 & 0 \\
\hline 222 & L. chuanxiong Hort & $\beta$-sesquiphellandrene & $20307-83-9$ & 204.39 & 23.68 & 1.89 & 0.06 & 5.14 & 0 & 0 \\
\hline 223 & L. chuanxiong Hort & $\begin{array}{l}\text { (4aS,7S,8aR)-7-isopropenyl-4a-methyl-1-methylen } \\
\text { edecalin }\end{array}$ & $18423-23-9$ & 204.39 & 23.65 & 1.83 & 0.08 & 4.81 & 0 & 0 \\
\hline 224 & L. chuanxiong Hort & artemisia triene & $29548-02-5$ & 136.26 & 42.1 & 1.84 & 0.02 & 3.24 & 0 & 0 \\
\hline 225 & L. chuanxiong Hort & betea-cubebene & $13744-15-5$ & 204.39 & 32.16 & 1.82 & 0.11 & 4.22 & 0 & 0 \\
\hline 226 & L. chuanxiong Hort & (1S,4E,8E,10R)-4,8,11,11-tetramethylbicyclo[8.1. & $24703-35-3$ & 204.39 & 21.69 & 1.86 & 0.08 & 4.7 & 0 & 0 \\
\hline
\end{tabular}




\begin{tabular}{|c|c|c|c|c|c|c|c|c|c|c|}
\hline & & 0]undeca-4,8-diene & & & & & & & & \\
\hline 227 & L. chuanxiong Hort & (Z)-ligustilide & $551-08-6$ & 188.24 & 53.72 & 1.3 & 0.07 & 3 & 0 & 2 \\
\hline 228 & L. chuanxiong Hort & chuanxiongol & $87421-30-5$ & 218.27 & 22.19 & 0.94 & 0.1 & 2.57 & 1 & 3 \\
\hline 229 & L. chuanxiong Hort & beta-asarone & $5273-86-9$ & 208.28 & 35.61 & 1.45 & 0.06 & 2.74 & 0 & 3 \\
\hline 230 & L. chuanxiong Hort & cis-piperitol & $16721-38-3$ & 154.28 & 43.26 & 1.27 & 0.03 & 2.66 & 1 & 1 \\
\hline 231 & L. chuanxiong Hort & $\begin{array}{l}(1 \mathrm{~S}, 4 \mathrm{R}, 5 \mathrm{R})-1 \text {-isopropyl-4-methyl-4-bicyclo[3.1.0] } \\
\text { hexanol }\end{array}$ & $546-79-2$ & 154.28 & 27.22 & 1.17 & 0.05 & 1.91 & 1 & 1 \\
\hline 232 & L. chuanxiong Hort & cnidilide & $3674-03-1$ & 194.3 & 77.55 & 1.21 & 0.07 & 2.97 & 0 & 2 \\
\hline 233 & L. chuanxiong Hort & 1,3,8-p-menthatriene & $18368-95-1$ & 134.24 & 35.86 & 1.86 & 0.02 & 3.25 & 0 & 0 \\
\hline 234 & L. chuanxiong Hort & cyclododecene & $1501-82-2$ & 166.34 & 47.89 & 1.84 & 0.03 & 5.03 & 0 & 0 \\
\hline 235 & L. chuanxiong Hort & cyclohexane,1,1,2,3-tetramethyl- & $6783-92-2$ & 140.3 & 48.08 & 1.77 & 0.03 & 3.7 & 0 & 0 \\
\hline 236 & L. chuanxiong Hort & 2,9-dimethyldecane & $1002-17-1$ & 170.38 & 9.93 & 1.82 & 0.02 & 5.44 & 0 & 0 \\
\hline 237 & L. chuanxiong Hort & $\begin{array}{l}(2 \mathrm{R}, 4 \mathrm{aR})-2 \text {-isopropenyl-4a,8-dimethyl-2,3,4,5,6,7- } \\
\text { hexahydro-1H-naphthalene }\end{array}$ & $17627-30-4$ & 204.39 & 22.13 & 1.86 & 0.08 & 4.95 & 0 & 0 \\
\hline 238 & L. chuanxiong Hort & 1,5,5-trimethyl-6-methylenecyclohexene & $514-95-4$ & 136.26 & 46.08 & 1.83 & 0.03 & 3.25 & 0 & 0 \\
\hline 239 & L. chuanxiong Hort & isobutyrophenone & $611-70-1$ & 148.22 & 80.37 & 1.46 & 0.03 & 2.7 & 0 & 1 \\
\hline 240 & L. chuanxiong Hort & myricanone & $32492-74-3$ & 356.45 & 40.6 & 0.67 & 0.51 & 4.1 & 2 & 5 \\
\hline 241 & L. chuanxiong Hort & neocnidilide & $4567-33-3$ & 194.3 & 83.83 & 1.23 & 0.07 & 3.37 & 0 & 2 \\
\hline 242 & L. chuanxiong Hort & octane & $31372-91-5$ & 114.26 & 29.72 & 1.78 & 0.01 & 4.02 & 0 & 0 \\
\hline 243 & L. chuanxiong Hort & p-cymen-8-ol & 1197-01-9 & 150.24 & 32.26 & 1.33 & 0.03 & 2.29 & 1 & 1 \\
\hline 244 & L. chuanxiong Hort & acetylphenylhydrazine & $114-83-0$ & 150.2 & 24.76 & 1.17 & 0.03 & 0.81 & 2 & 3 \\
\hline 245 & L. chuanxiong Hort & perlolyrine & $29700-20-7$ & 264.3 & 65.95 & 0.88 & 0.27 & 3.2 & 2 & 3 \\
\hline 246 & L. chuanxiong Hort & 3beta-hydroxypregn-5-ene-20-one & $145-13-1$ & 316.53 & 14.07 & 0.69 & 0.43 & 3.49 & 1 & 2 \\
\hline 247 & L. chuanxiong Hort & sedanoic-acid & $6697-07-0$ & 210.3 & 44.69 & 0.37 & 0.06 & 2.58 & 1 & 3 \\
\hline 248 & L. chuanxiong Hort & senkyunolide C & $63038-10-8$ & 204.24 & 46.8 & 0.87 & 0.08 & 2.74 & 1 & 3 \\
\hline 249 & L. chuanxiong Hort & senkyunolide D & $93236-67-0$ & 222.26 & 79.13 & 0.12 & 0.1 & 1.8 & 1 & 4 \\
\hline 250 & L. chuanxiong Hort & senkyunolide E & $91652-78-7$ & 204.24 & 34.4 & 0.55 & 0.08 & 1.9 & 1 & 3 \\
\hline 251 & L. chuanxiong Hort & senkyunolide F & $94530-84-4$ & 206.26 & 40.35 & 0.61 & 0.08 & 1.84 & 1 & 3 \\
\hline
\end{tabular}




\begin{tabular}{|c|c|c|c|c|c|c|c|c|c|c|}
\hline 252 & L. chuanxiong Hort & senkyunolide J & $94530-86-6$ & 226.3 & 21.14 & 0.02 & 0.1 & 1.57 & 2 & 4 \\
\hline 253 & L. chuanxiong Hort & senkyunolide L & $114586-51-5$ & 242.72 & 29.64 & 0.63 & 0.09 & 2.52 & 1 & 3 \\
\hline 254 & L. chuanxiong Hort & senkyunolide $\mathrm{S}$ & N/A & 240.33 & 20.61 & -0.46 & 0.11 & 0.44 & 3 & 4 \\
\hline 255 & L. chuanxiong Hort & 1-acetyl-beta-carboline & $50892-83-6$ & 210.25 & 67.12 & 1.18 & 0.13 & 2.34 & 1 & 2 \\
\hline 256 & L. chuanxiong Hort & senkyunone & $142182-61-4$ & 326.52 & 47.66 & 1.15 & 0.24 & 6.32 & 0 & 2 \\
\hline 257 & L. chuanxiong Hort & sinapic acid & $530-59-6$ & 224.23 & 64.15 & 0.48 & 0.08 & 1.6 & 2 & 5 \\
\hline 258 & L. chuanxiong Hort & $\begin{array}{l}\text { 1H-cycloprop(e)azulen-7-ol, } \\
\text { decahydro-1,1,7-trimethyl-4-methylene-, } \\
\text { (1aR-(1aalpha,4aalpha,7beta,7abeta,7balpha)) }\end{array}$ & $6750-60-3$ & 220.39 & 82.33 & 1.37 & 0.12 & 3.01 & 1 & 1 \\
\hline 259 & L. chuanxiong Hort & trans-2-nonen-1-ol & $22104-79-6$ & 142.27 & 19.96 & 1.17 & 0.02 & 2.94 & 1 & 1 \\
\hline 260 & L. chuanxiong Hort & $\mathrm{N}, \mathrm{N}$-dimethylmethanamine & $175-50-3$ & 59.13 & 59.98 & 1.78 & 0 & 0.32 & 0 & 1 \\
\hline 261 & L. chuanxiong Hort & wallichilide & $93236-64-7$ & 412.57 & 42.31 & 0.82 & 0.71 & 4.82 & 0 & 5 \\
\hline 262 & L. chuanxiong Hort & xiongterpene & $\mathrm{N} / \mathrm{A}$ & 574.87 & 23.77 & 0.44 & 0.42 & 8.12 & 2 & 5 \\
\hline 263 & L. chuanxiong Hort & 1-octanol,2,7-dimethyl- & N/A & 158.32 & 24.43 & 1.25 & 0.02 & 3.37 & 1 & 1 \\
\hline 264 & L. chuanxiong Hort & 1-terpineol & $586-82-3$ & 154.28 & 49.83 & 1.24 & 0.03 & 2.42 & 1 & 1 \\
\hline 265 & L. chuanxiong Hort & 1-beta-ethylacrylate-7-aldehyde-beta-carboline & N/A & 294.33 & 28.53 & 0.45 & 0.31 & 3.03 & 1 & 4 \\
\hline 266 & L. chuanxiong Hort & 2-propionylfuran & $3194-15-8$ & 124.15 & 63.12 & 1.21 & 0.02 & 1.63 & 0 & 2 \\
\hline 267 & L. chuanxiong Hort & 1-phenylpropan-1-one & $93-55-0$ & 134.19 & 60.17 & 1.45 & 0.02 & 2.24 & 0 & 1 \\
\hline 268 & L. chuanxiong Hort & 2,2,3-trimethylcyclopent-3-ene-1-carboxaldehyde & $33843-21-9$ & 138.23 & 42.64 & 1.31 & 0.03 & 1.95 & 0 & 1 \\
\hline 269 & L. chuanxiong Hort & methyl 2-pentanoylbenzoate & $\mathrm{N} / \mathrm{A}$ & 220.29 & 69.28 & 0.91 & 0.07 & 3.01 & 0 & 3 \\
\hline 270 & L. chuanxiong Hort & isoheptane & $591-76-4$ & 100.23 & 59.94 & 1.81 & 0.01 & 3.36 & 0 & 0 \\
\hline 271 & L. chuanxiong Hort & methyl furan-2-carboxylate & $611-13-2$ & 126.12 & 49.41 & 1.12 & 0.02 & 1.08 & 0 & 3 \\
\hline 272 & L. chuanxiong Hort & 2-methyl-1-phenylpropene & $768-49-0$ & 132.22 & 20.17 & 1.89 & 0.02 & 3.23 & 0 & 0 \\
\hline 273 & L. chuanxiong Hort & 2-methyl-5-(1-methylene)-1,3-cyclohexadiene & $\mathrm{N} / \mathrm{A}$ & 106.18 & 39.91 & 1.83 & 0.03 & 2.17 & 0 & 0 \\
\hline 274 & L. chuanxiong Hort & $(4 \mathrm{~S}, 6 \mathrm{~S})$-cis-carveol & $99-48-9$ & 152.26 & 32.5 & 1.22 & 0.03 & 2.4 & 1 & 1 \\
\hline 275 & L. chuanxiong Hort & 2-methylbenzoxazol & $95-21-6$ & 133.16 & 65.25 & 1.31 & 0.03 & 1.62 & 0 & 2 \\
\hline 276 & L. chuanxiong Hort & (5S,6R)-5,6-dimethyltetrahydropyran-2-one & $24405-16-1$ & 128.19 & 48.07 & 1.15 & 0.02 & 1.43 & 0 & 2 \\
\hline 277 & L. chuanxiong Hort & 3(S)-3-butyl-4,5-dihydrophthalide & $\mathrm{N} / \mathrm{A}$ & 194.3 & 25.76 & 1.28 & 0.07 & 3.64 & 0 & 2 \\
\hline
\end{tabular}




\begin{tabular}{|c|c|c|c|c|c|c|c|c|c|c|}
\hline 278 & L. chuanxiong Hort & 3,4-epoxy-2,2,7,7-tetramethyl-octane & $\mathrm{N} / \mathrm{A}$ & 184.36 & 66.87 & 1.56 & 0.05 & 3.64 & 0 & 1 \\
\hline 279 & L. chuanxiong Hort & 3-cyclohexen-1-ol & $822-66-2$ & 98.16 & 70.57 & 1.14 & 0.01 & 1.06 & 1 & 1 \\
\hline 280 & L. chuanxiong Hort & methyl 3-furoate & $1334-76-5$ & 126.12 & 77.82 & 1.03 & 0.02 & 0.79 & 0 & 3 \\
\hline 281 & L. chuanxiong Hort & trans-piperitol & $16721-39-4$ & 154.28 & 47.83 & 1.19 & 0.03 & 2.66 & 1 & 1 \\
\hline 282 & L. chuanxiong Hort & 4,7-dihydroxy-3-butylphthalide & N/A & 222.26 & 106.09 & 0.69 & 0.1 & 2.72 & 2 & 4 \\
\hline 283 & L. chuanxiong Hort & 4-iodoindoline & 939759-03-2 & 245.07 & 26.88 & 1.79 & 0.03 & 2.29 & 1 & 1 \\
\hline 284 & L. chuanxiong Hort & 4-octanone & $589-63-9$ & 128.24 & 19.37 & 1.37 & 0.01 & 2.46 & 0 & 1 \\
\hline 285 & L. chuanxiong Hort & 4-hydroxy-3-butylphthalide & N/A & 206.26 & 70.31 & 0.9 & 0.08 & 2.99 & 1 & 3 \\
\hline 286 & L. chuanxiong Hort & (-)-spathulenol & $77171-55-2$ & 220.39 & 25.82 & 1.45 & 0.12 & 3.01 & 1 & 1 \\
\hline 287 & L. chuanxiong Hort & 5-propyl-2-thiouracil & $2954-52-1$ & 170.26 & 77.6 & 0.93 & 0.03 & 1.25 & 2 & 3 \\
\hline 288 & L. chuanxiong Hort & (6R)-6-butylcyclohepta-1,4-diene & $33156-91-1$ & 150.29 & 31.69 & 1.85 & 0.02 & 3.93 & 0 & 0 \\
\hline 289 & L. chuanxiong Hort & $\begin{array}{l}\text { 7-oxabicyclo-2.2.1-heptane,1-methyl-4-[1-methyle } \\
\text { thyl] }\end{array}$ & $470-67-7$ & 154.28 & 60.92 & 1.53 & 0.04 & 2.28 & 0 & 1 \\
\hline 290 & L. chuanxiong Hort & aromadendrene oxide 2 & $85710-39-0$ & 220.39 & 65.1 & 1.56 & 0.14 & 3.08 & 0 & 1 \\
\hline 291 & L. chuanxiong Hort & amylbenzene & $27458-20-4$ & 148.27 & 34.34 & 1.88 & 0.03 & 4.14 & 0 & 0 \\
\hline 292 & L. chuanxiong Hort & dimethyl D-malate & $70681-41-3$ & 162.16 & 11.47 & 0.11 & 0.03 & -0.45 & 1 & 5 \\
\hline 293 & L. chuanxiong Hort & dl-3n-butylphthalide & $6066-49-5$ & 190.26 & 47.9 & 1.3 & 0.07 & 3.25 & 0 & 2 \\
\hline 294 & L. chuanxiong Hort & cedrene & $469-61-4$ & 204.39 & 51.14 & 1.82 & 0.11 & 4.18 & 0 & 0 \\
\hline 295 & L. chuanxiong Hort & carotol & $465-28-1$ & 222.41 & 149.03 & 1.46 & 0.09 & 3.94 & 1 & 1 \\
\hline 296 & L. chuanxiong Hort & coniferylfcrulate & $63644-62-2$ & 356.4 & 4.54 & 0.67 & 0.39 & 3.64 & 2 & 6 \\
\hline 297 & L. chuanxiong Hort & cerulignol & $2785-87-7$ & 166.24 & 62.43 & 1.42 & 0.04 & 2.95 & 1 & 2 \\
\hline 298 & L. chuanxiong Hort & $\begin{array}{l}\text { decahydro-1,6-bis(methylene)-4-(1-methylethyl)-n } \\
\text { aphthalene }\end{array}$ & $54932-90-0$ & 204.39 & 28.34 & 1.84 & 0.08 & 4.85 & 0 & 0 \\
\hline 299 & L. chuanxiong Hort & hexaphenone & $942-92-7$ & 176.28 & 19.88 & 1.49 & 0.04 & 3.61 & 0 & 1 \\
\hline 300 & L. chuanxiong Hort & (E,E)-1,3,5-undecatriene & $19883-29-5$ & 150.29 & 34.61 & 1.84 & 0.02 & 4.11 & 0 & 0 \\
\hline 301 & L. chuanxiong Hort & heptan & $142-82-5$ & 100.23 & 41.8 & 1.77 & 0 & 3.57 & 0 & 0 \\
\hline 302 & L. chuanxiong Hort & L-valyl-L-valinc-achydride & $350702-73-7$ & 214.35 & 40.18 & -0.15 & 0.05 & 0.47 & 4 & 4 \\
\hline 303 & L. chuanxiong Hort & levistolide A & $88182-33-6$ & 380.52 & 9.96 & 0.96 & 0.82 & 4.97 & 0 & 4 \\
\hline
\end{tabular}




\begin{tabular}{|c|c|c|c|c|c|c|c|c|c|c|}
\hline 304 & L. chuanxiong Hort & cis-ligustilide & $81944-08-3$ & 190.26 & 51.3 & 1.3 & 0.07 & 2.94 & 0 & 2 \\
\hline 305 & L. chuanxiong Hort & tetramethylpyrazine & $87396-75-6$ & 136.22 & 20.01 & 1.19 & 0.03 & 0.66 & 0 & 2 \\
\hline 306 & L. chuanxiong Hort & methyl (Z)-octadec-9-enoate & $139152-82-2$ & 296.55 & 31.9 & 1.39 & 0.16 & 7.09 & 0 & 2 \\
\hline 307 & L. chuanxiong Hort & $(9 Z, 12 Z)$-octadeca-9,12-dien-1-ol & $506-43-4$ & 266.52 & 37.76 & 1.41 & 0.12 & 6.47 & 1 & 1 \\
\hline 308 & L. chuanxiong Hort & octatriacontane & $7194-85-6$ & 535.16 & 7.91 & 1.99 & 0.37 & 17.71 & 0 & 0 \\
\hline 309 & L. chuanxiong Hort & (2-amylphenyl)methanol & $\mathrm{N} / \mathrm{A}$ & 178.3 & 55.59 & 1.26 & 0.04 & 3.54 & 1 & 1 \\
\hline 310 & L. chuanxiong Hort & $\begin{array}{l}\text { (3S,3aR)-3-butyl-3a,4,5,6-tetrahydro-3H-2-benzof } \\
\text { uran-1-one }\end{array}$ & $4567-33-3$ & 194.3 & 65.03 & 1.25 & 0.07 & 3.37 & 0 & 2 \\
\hline 311 & L. chuanxiong Hort & senkyunolide A & $63038-10-8$ & 192.28 & 26.56 & 1.3 & 0.07 & 3.19 & 0 & 2 \\
\hline 312 & L. chuanxiong Hort & senkyunolide G & $94530-85-5$ & 208.28 & 39.52 & 0.63 & 0.08 & 2.54 & 1 & 3 \\
\hline 313 & L. chuanxiong Hort & $\begin{array}{l}\text { (3Z,6S,7S)-3-butylidene-6,7-dihydroxy-4,5,6,7-tetr } \\
\text { ahydroisobenzofuran-1-one }\end{array}$ & $94596-27-7$ & 224.28 & 26.78 & 0 & 0.1 & 1.32 & 2 & 4 \\
\hline 314 & L. chuanxiong Hort & germacrene D & $23986-74-5$ & 204.39 & 19.22 & 1.83 & 0.06 & 5.14 & 0 & 0 \\
\hline 315 & L. chuanxiong Hort & falcarindiol & $225110-25-8$ & 260.41 & 39.30 & 0.37 & 0.11 & 4.71 & 2 & 2 \\
\hline
\end{tabular}

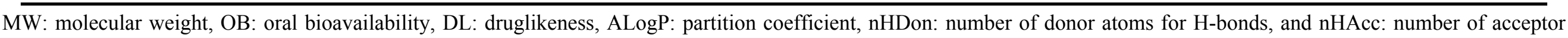
atoms for H-bonds. 

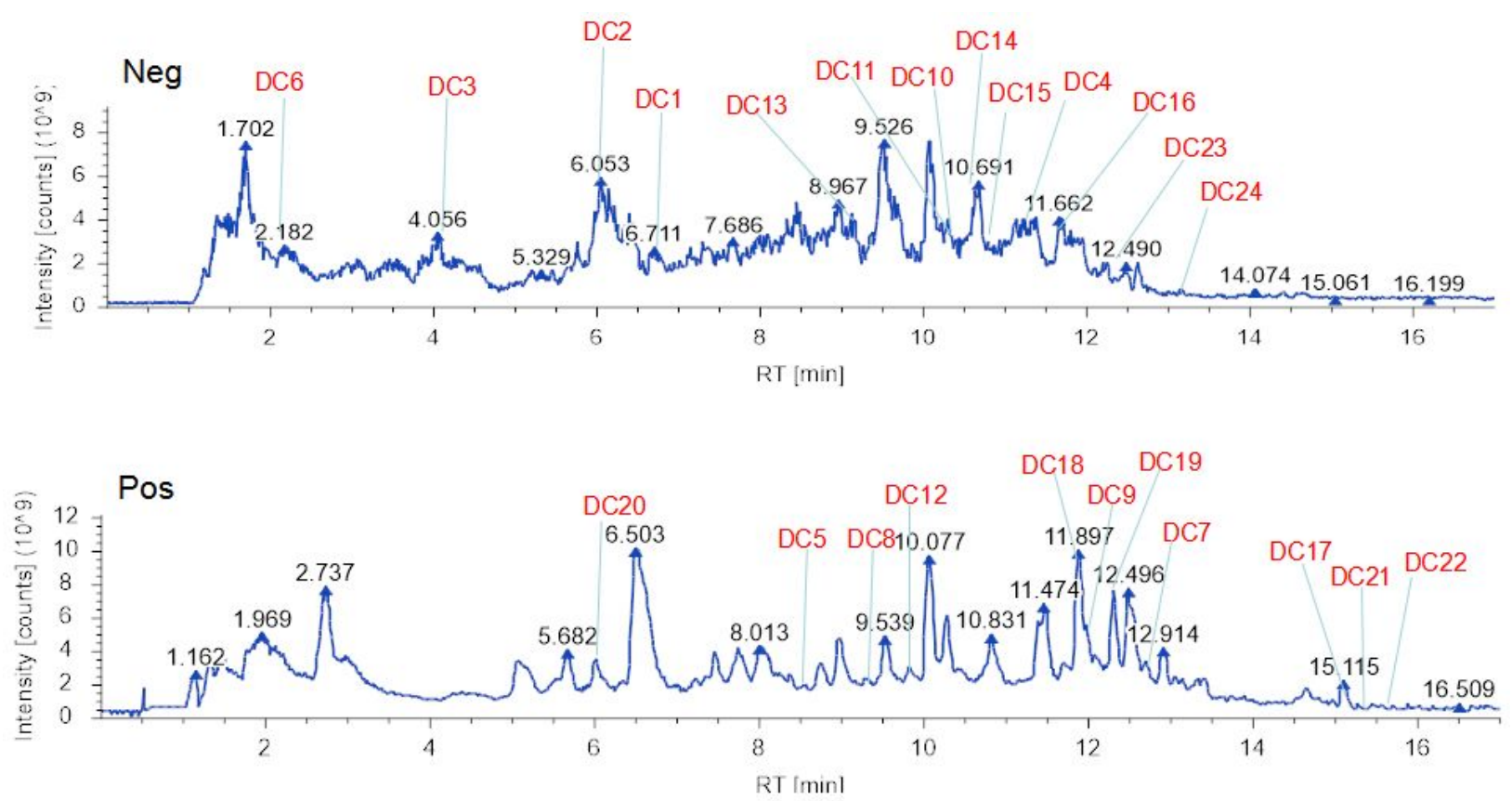

Figure S1 The total ion chromatograms (TIC) of the active compounds of DC by ultra high-performance liquid chromatography Q-Exactive HF mass spectrometry (UHPLC-Q-Exactive HF/MS) in negative and positive ion modes).

\section{UHPLC-Q-Exactive HF/MS Analysis of DC}

DC extract $(100 \mathrm{mg})$ was placed in an EP tube, and added $500 \mu \mathrm{L}$ of $80 \%$ methanol aqueous solution. The mixture was vortexed and shaked, and centrifuged for 20 min at 15000 $g$ under $4{ }^{\circ} \mathrm{C}$. The supernatant was diluted with mass spectrometry water to a methanol content of $53 \%$, and then centrifuged for another $20 \mathrm{~min}$ at $15000 \mathrm{~g}$ under $4{ }^{\circ} \mathrm{C}$ to obtain the supernatant for LC-MS analysis. Equal amount of supernatant was taken from each processed samples and mixed as quality control (QC) samples. The experimental sample was replaced by $53 \%$ methanol aqueous solution to obtain blank sample, and the pretreatment process was the same as that of the experimental sample.

The analysis of DC was performed on a Vanquish UHPLC system (Thermo, Massachusetts, America) equipped with an Hypesil Gold column (Thermo, Massachusetts, America; $100 \times 2.1 \mathrm{~m} \mathrm{~m}, 1.9 \mu \mathrm{m})$. The mobile phase A of positive ion mode was composed of $0.1 \%$ formic acid, and that of negative ion mode was $5 \mathrm{mmol} / \mathrm{L}$ ammonium acetate. The mobile phase $\mathrm{B}$ of these two ion modes were both methanol. The elution conditions of UHPLC were as follows: 0-1.5 min, 98-98\% A; 1.5-12 min, 98-0\% A; $12-14 \mathrm{~min}, 0-0 \% \mathrm{~A}$; 
14-14.1 $\mathrm{min}, 0-98 \% \mathrm{~A} ; 14.1-17 \mathrm{~min}, 98-98 \% \mathrm{~A}$. The flow rate was $0.2 \mathrm{~mL} / \mathrm{min}$, and the column temperature was $40{ }^{\circ} \mathrm{C}$ during the analysis process. A Q-Exactive $\mathrm{HF}$ mass spectrometer (Thermo, Massachusetts, America) was used to identify the constituents. The ESI source conditions were set as follows: spray voltage, $3.2 \mathrm{kV}$; sheath gas flow rate, 40arb; aux gasflow rate, $10 \mathrm{arb}$; capillary temp, $320^{\circ} \mathrm{C}$; polarity, positive and negative.

The raw data files generated by UHPLC-Q-Exactive HF/MS were processed using the Compound Discoverer 3.1 (CD3.1, ThermoFisher) to perform peak alignment, peak picking, and quantitation for each metabolite. The main parameterswere set as follows: retention time tolerance, 0.2 minutes; actual mass tolerance, 5ppm; signal intensity tolerance, $30 \%$; signal/noise ratio, 3; and minimum intensity, et al. After that, peak intensities were normalized to the total spectral intensity. The normalized data was used to predict the molecular formula based on additive ions, molecular ion peaks and fragment ions. And then peaks were matched with the mzCloud (https://www.mzcloud.org/), mzVault and MassList database to obtain the accurate qualitative and relative quantitative results. Statistical analyses were performed using the statistical software R (R version R-3.4.3), Python (Python 2.7.6 version) and CentOS (CentOS release 6.6), When data were not normally distributed, normal transformations were attempted using of area normalization method. 